\title{
Segurança do paciente em tratamento dialítico
}

\author{
Patient safety in dialysis treatments
}

Resumo O estudo teve como objetivo analisar a produção científica com a temática segurança do paciente em tratamento dialítico; levantar os principais eventos que comprometem a segurança do paciente nesse tratamento; e identificar ações de segurança desenvolvidas pelos serviços de saúde para minimizar estes eventos. Trata-se de uma revisão integrativa, cuja coleta de dados ocorreu na base de dados Biblioteca Virtual em Saúde. Foram incluídos na revisão 14 artigos, e a análise dos dados se deu por meio da análise de conteúdo. Nos resultados emergiram quatro categorias: Implementação de ações para segurança do paciente em diálise; Clima/Cultura de segurança em pacientes em diálise; Conhecimento dos profissionais de saúde sobre segurança do paciente em diálise; e Incidentes/Eventos Adversos em diálise. Os eventos adversos identificados foram: deslocamento da agulha em punções de fístula arteriovenosa, infecções, disfunção do acesso, coagulação do sistema extracorpóreo e quedas. Sendo as ações para diminuição desses eventos: educação continuada, definição de diretrizes e políticas, adoção de ações não punitivas com incentivo à notificação dos eventos adversos, implementação de listas de tarefas e responsabilidades, checklist de verificação. A unidade de diálise é um ambiente propício para o acontecimento de eventos adversos, necessitando assim, da fomentação de estratégias que identifiquem e previnam riscos.

Palavras-chave: Diálise. Segurança do paciente. Eventos adVERSOS.

Abstract The objective of this study was to analyze the scientific production with the theme of patient safety in dialysis treatment; to raise the main events that compromise patient safety in this treatment; and identify safety actions developed by health services. It is an integrative review whose data collection took place in the Virtual Health Library database. A total of 14 articles were included in the review, and data analysis was done through content analysis. We found four categories: Implementation of actions for patient safety in dialysis, Climate / safety culture in dialysis patients, Knowledge of health professionals about patient safety in dialysis and Incident / Adverse Events in dialysis. The adverse events identified were: needle displacement in AVF punctures, infections, access dysfunction, extracorporeal system coagulation and falls. The actions to reduce these events are: continuing education, defi-

Maria Rosa Silva Lemos Universidade do Estado da Bahia (UNEB), Salvador/BH - Brasil. Edenise Maria Santos da Silva BatalHa

Universidade do Estado da Bahia (UNEB), Salvador/BH - Brasil. nition of guidelines and policies, adoption of non-punitive actions with incentive to report adverse events, implementation of task lists and responsibilities, verification checklist. The dialysis unit is an environment conducive to the occurrence of adverse events, thus requiring the development of strategies that identify and prevent risks.

Key-words: Dialysis. Patient SAfety. Adverse events. 


\section{INTRODUÇÃO}

A Insuficiência Renal Crônica (IRC) é a perda da função renal de forma lenta e progressiva, de modo que o organismo não consegue manter o equilíbrio metabólico e hidroeletrolítico, sendo necessária a utilização de uma terapia renal substitutiva. ${ }^{1}$

$\mathrm{O}$ quantitativo de indivíduos que necessitam de tratamento dialítico vem apresentando um crescimento exponencial. Os dados demonstram que o número estimado de pacientes em tratamento dialítico no Brasil nos últimos cinco anos sofreu um aumento de 31,5 mil pacientes, sendo que, em 2011, o número estimado de pacientes em terapia renal substitutiva era de 91.314 pacientes e, em 2016, um total de 122.825 pacientes. $^{2}$

As modalidades de terapia renal substitutiva disponíveis são: i) a hemodiálise; ii) a diálise peritoneal; e iii) o transplante renal.

A hemodiálise consiste na filtração do sangue por meio de uma máquina em que é utilizado um dialisador, que serve como membrana semipermeável, onde são removidos as toxinas e resíduos nitrogenados do sangue do paciente. ${ }^{1}$

A diálise peritoneal utiliza o peritônio como membrana para a realização da filtração das toxinas e eliminação do excesso de líquido, implantando-se um cateter no abdômen, por onde são infundidos líquidos de diálise que permanecem na cavidade peritoneal onde acontecem as trocas por difusão e o excesso de líquido e toxinas migra para o líquido de diálise, que é drenado por meio dessa mesma via. ${ }^{3}$

O transplante renal consiste no implante de um órgão saudável de uma pessoa viva ou morta por meio de uma cirurgia, passando esse rim implantado a exercer as funções de filtração e eliminação de líquidos e toxinas. No transplante intervivos, além da compatibilidade, é muito importante avaliar o grau de parentesco e a afetividade entre doador e receptor. ${ }^{4} \mathrm{O}$ transplante renal com doador morto ainda é realizado em pequena escala, devido à desproporção entre a demanda de órgãos para transplante e o número de transplantes efetivados. Essa desproporção está associada a grandes problemas como: falhas no processo de reconhecimento da morte encefálica, na abordagem da família durante as entrevistas, manutenção clínica do doador morto e de contraindicações mal atribuídas. ${ }^{5}$

Diante das diversas modalidades de terapias renais substitutivas, é notória a grande manipulação e exposição do indivíduo que não tem suas funções renais em níveis compatíveis com a vida, portanto, faz-se necessário a instituição de ações que garantam a segurança do paciente nos serviços e diminuam a probabilidade de complicações.

Segurança do Paciente vem sendo foco de discussões nacionais e internacionais desde a criação da Aliança Mundial para Segurança do Paciente, criada em 2004, pela Organização Mundial de Saúde.

Para legitimação dos esforços acerca da segurança do paciente, o Ministério da Saúde traz, na Portaria $n^{\circ}$. 529, de $1^{\circ}$. de abril de 2013, a instituição do Programa Nacional de Segurança do Paciente (PNSP), que visa "prevenir, monitorar e reduzir a incidência de EAs nos atendimentos prestados, promovendo melhorias relacionadas à segurança do paciente e a qualidade em serviços de saúde do País".

Ainda na referida Portaria, Segurança 
do Paciente é conceituada como redução a um mínimo aceitável do risco de dano desnecessário associado ao cuidado de saúde. ${ }^{6}$

Antes do acontecimento da Tragédia de Caruaru, como assim ficou conhecida no ano 1996, quando morreram $80 \%$ dos pacientes da unidade por hepatite tóxica, uma infecção causada pela bactéria microcistina presente na água contaminada durante o tratamento dialítico, não existiam políticas para os serviços de diálise e a vigilância atuava de forma ineficaz. ${ }^{7}$

Após esse fato, discussões e mudanças foram fomentadas, dando origem à Portaria 2.042 de 11 de outubro de 1996, estabelecendo o regulamento técnico para o funcionamento dos serviços de terapia renal, sendo reformulada em 2004, dando origem à Resolução de Diretoria Colegiada - RDC 154, que institui que todo serviço de diálise deve implantar e implementar um Programa de Controle e Prevenção de Infecção e de Eventos Adversos (PCPIEA). Em 13 de março de 2014, foi publicada a RDC nº. 11, que reforça e estabelece novos requisitos de boas práticas para o funcionamento dos serviços de diálise. ${ }^{7}$

Devido à necessidade do paciente renal crônico submeter-se com grande frequência a tratamentos de saúde, portanto, estando exposto a riscos evitáveis constantemente e sendo de grande relevância a identificação dos eventos adversos e a adoção de ações que culminem na prestação de uma assistência de qualidade centrada na segurança do paciente, foi elaborada a seguinte pergunta norteadora para este estudo: Como se caracteriza a produção científica em relação à segurança do paciente em tratamento de diálise nos serviços de saúde?

Assim, o objetivo geral do presente estudo foi analisar a produção científica que aborda a temática da segurança do paciente em tratamento de diálise nos serviços de saúde, para que, aprofundando-se nesta, pudéssemos atingir os objetivos específicos, cujos quais foram: levantar os principais eventos que comprometem a segurança do paciente em tratamentos de diálise e identificar as ações de segurança do paciente desenvolvidas pelos serviços de saúde.

\section{Métodos}

A metodologia utilizada foi a de revisão integrativa que, conforme afirmam Souza et al. ${ }^{8}$ e Mendes et al., ${ }^{9}$ é um método que reúne o conhecimento científico, proporcionando uma prática baseada em evidências.

Ainda, conforme Souza et al. $^{8}$ e Mendes et al., ${ }^{9}$ a revisão integrativa de literatura é composta por seis etapas, sendo elas: 1) Delimitação do tema com a formulação da pergunta norteadora que irá determinar quais serão os estudos incluídos, os meios adotados para a identificação e as informações coletadas de cada estudo selecionado; 2) Amostragem ou busca na literatura, a qual deve ser ampla e diversificada; 3) $\mathrm{Ca}$ tegorização dos estudos, momento em que o revisor, por meio de um instrumento previamente elaborado, irá organizar e sumarizar as informações chaves; 4) Análise crítica dos estudos incluídos, equivalente à análise de dados em uma pesquisa convencional; 5) Interpretação dos resultados, em que após a interpretação e síntese dos dados obtidos por intermédio da análise dos artigos é rea- 
lizada uma comparação com o referencial teórico; 6) Apresentação da revisão integrativa com descrição das etapas percorridas e apresentação dos principais resultados.

Os dados foram coletados por intermédio da Biblioteca Virtual em Saúde BVS, com acesso aos bancos de dados LILACS - Literatura Latino-Americana e do Caribe em Ciências da Saúde e ao SCIELO - Scientific Eletronic Library Online e outros bancos de dados inseridos na BVS/BIREME, que apresentassem artigos que se enquadrassem nos critérios de inclusão desta revisão.

Os descritores adotados foram: segurança do paciente, diálise, eventos adversos, incidentes e seus análogos nas línguas inglesa e espanhola. Os descritores empregados fazem parte dos Descritores em Ciências da Saúde (DeCS) com a opção "termo exato" e foi utilizado como operador booleano "AND".

Os critérios adotados para a seleção dos artigos foram: artigos originais; disponíveis na íntegra; publicados nas línguas inglesa, portuguesa e espanhola no período de janeiro de 2004 até setembro de 2017, que continham em seus títulos e/ou resumos os seguintes descritores: doença renal crônica, diálise e hemodiálise associados à segurança do paciente e eventos adversos e destacando aqueles que respondiam aos objetivos do estudo.

A escolha do marco temporal a partir de 2004 se deu pelo lançamento, neste ano, da Aliança Mundial para Segurança do Paciente, que adota medidas de melhoria no atendimento ao paciente e aumenta a qualidade dos serviços de saúde. E o surgimento da RDC 154, que institui que todo serviço de diálise deve implantar e implementar um programa de Controle e Prevenção de Infec- ção e de Eventos Adversos (PCPIEA).

Os critérios de exclusão foram: artigos de revisão de literatura, que tinham como tratamento dialítico a diálise realizada em domicílio.

Como método de organização e tabulação dos dados, foi construído um instrumento de coleta dessas informações, contendo: título, autores, ano de publicação, periódico, local de publicação, principais resultados e conclusões.

A análise dos dados se deu por meio da técnica de análise de conteúdo temática ancorada em Bardin. ${ }^{10}$

\section{RESUltados}

Os resultados estão exibidos a seguir, segundo a caracterização dos artigos incluídos na pesquisa, apresentação da sumarização dos artigos conforme caracterização, principais resultados e conclusões.

A partir do cruzamento dos descritores "safety patient" AND "dialysis", "adverse events" AND "dialysis" e "incidents" AND "dialysis" no banco de dados utilizados com os filtros de idioma, período do estudo e disponibilidade dos artigos, foi encontrada a totalidade de 307 artigos. Após a leitura dos títulos, resumos e palavras-chave, além da aplicação dos critérios de inclusão e exclusão determinados na pesquisa, foram excluídos 293 artigos, sendo 23 por duplicidade e 270 por não atenderem aos objetivos e/ou critérios da pesquisa. Restando para a análise 14 artigos.

O Gráfico 1 a seguir apresenta a distribuição dos artigos por ano e localidade. 
GRÁFICO 1. Distribuição dos artigos por ano e localidade de publicação.

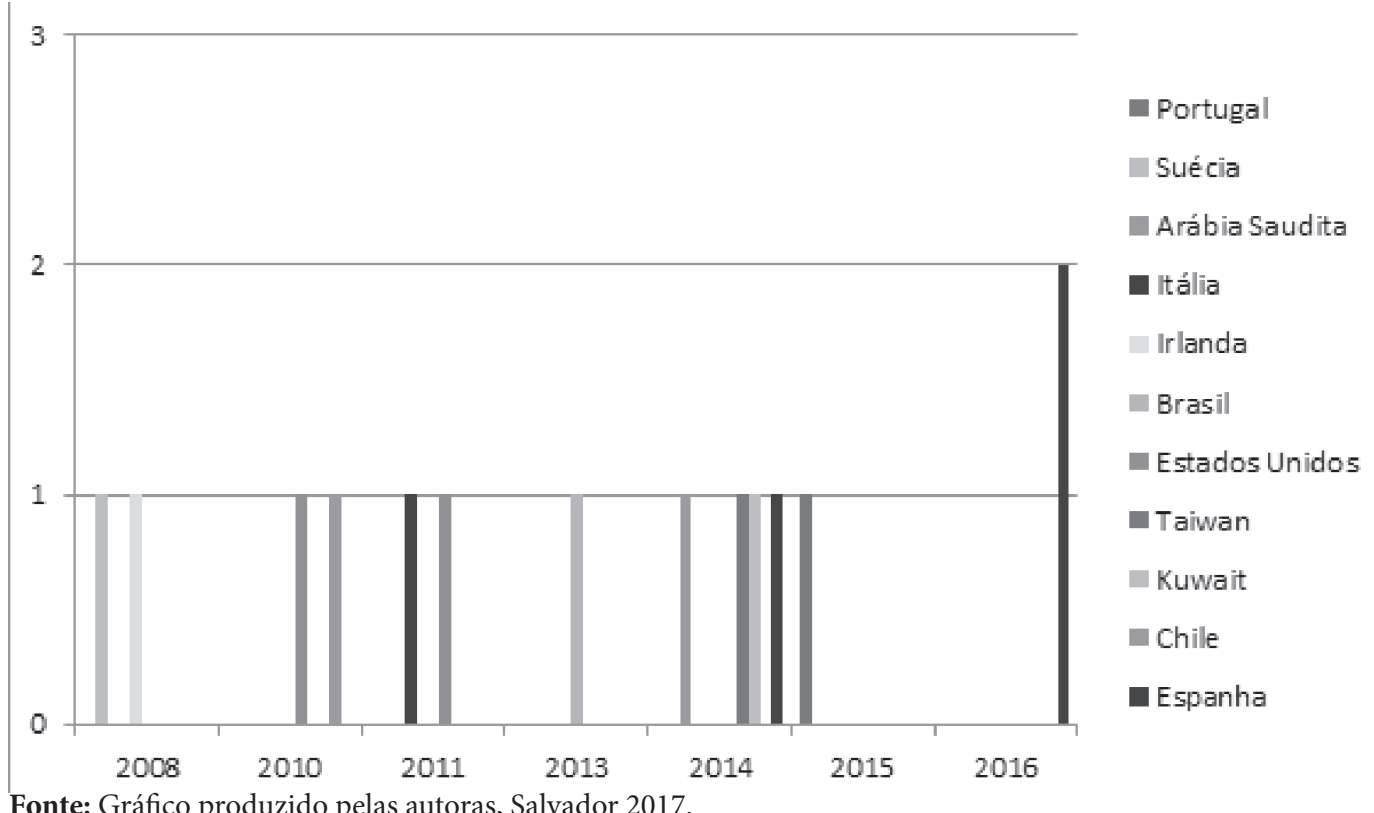

Fonte: Gráfico produzido pelas autoras, Salvador 2017.

Já o quadro a seguir traz a sumarização dos artigos, conforme caracterização, principais resultados e conclusões.

QUADRO 1. Sumarização dos artigos, conforme caracterização, principais resultados e conclusões.

\begin{tabular}{|l|l|l|}
\hline Artigo & $\begin{array}{l}\text { Autor e Ano/Periódico/ } \\
\text { Local de publicação }\end{array}$ & Principais resultados e conclusões \\
\hline $\begin{array}{l}\text { A1-Implementation } \\
\text { of a quality and } \\
\text { safety checklist for } \\
\text { haemodialysis sessions. }\end{array}$ & MARCELLI, D. et al., & $\begin{array}{l}\text { Foram identificadas quatro questões que impactam sobre a qualidade } \\
\text { e a segurança da diálise como: os atrasos no tempo de conexão; } \\
\text { tempo de tratamento incompleto; não realização do peso corporal } \\
\text { final e não atingir a um Kt/V de pelo menos 1,4. Listas de verificação } \\
\text { aumentam a qualidade global e a segurança do tratamento. }\end{array}$ \\
\hline $\begin{array}{l}\text { A2-A new safety device } \\
\text { for hemodialysis. }\end{array}$ & $\begin{array}{l}\text { AHLMÉN, J. et } \\
\text { al., 2008/ Journal } \\
\text { Compilation/ Suécia. }\end{array}$ & $\begin{array}{l}\text { Foram realizados 213 testes com boa aceitação do dispositivo } \\
\text { pelas enfermeiras responsáveis pelo teste. Em situações em que a } \\
\text { supervisão durante uma sessão de diálise pode ser insuficiente, o } \\
\text { dispositivo pode salvar vidas. }\end{array}$ \\
\hline $\begin{array}{l}\text { A3-Safety climate in } \\
\text { dialysis centers in Saudi } \\
\text { Arabia: a multicenter } \\
\text { study. }\end{array}$ & $\begin{array}{l}\text { TAHER, S. et al, 2014/ } \\
\text { tournal of Patient Safe- }\end{array}$ & $\begin{array}{l}\text { A taxa de concordância geral foi de 73,8\%, com mais enfermeiros } \\
\text { do que médicos concordando que o clima de segurança prevalece } \\
\text { nos centros de diálise. Os entrevistados perceberam um maior } \\
\text { compromisso com a segurança dos líderes da área clínica do } \\
\text { que com os líderes da organização. A alta administração precisa } \\
\text { transmitir seu compromisso com a segurança de forma mais } \\
\text { eficaz, especialmente para os médicos, e abrir canais de fácil } \\
\text { acesso para a comunicação sobre segurança. }\end{array}$ \\
\hline
\end{tabular}




\begin{tabular}{|c|c|c|}
\hline $\begin{array}{l}\text { A4-What causes an } \\
\text { improved safety climate } \\
\text { among the staff of a } \\
\text { dialysis unit? Report of } \\
\text { an evaluation in a large } \\
\text { network. }\end{array}$ & 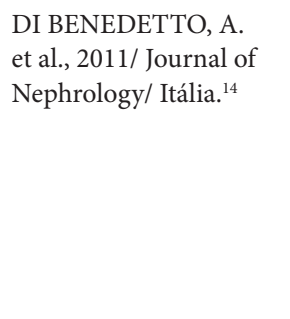 & $\begin{array}{l}\text { A média do clima de segurança foi de } 81,9 \% \text {. Com pouca } \\
\text { diferença dos diretores médicos e chefia de enfermagem, mas } \\
\text { diferença relativa entre diretores, médicos e enfermeiros de } \\
\text { pessoal. O pessoal da linha de frente mostrou significativamente } \\
\text { menor pontuação relativa ao manejo adequado de erros médicos e } \\
\text { a sensação de ser encorajados a relatar preocupações de segurança } \\
\text { que possam ter. Promover a comunicação e a implementação de } \\
\text { programas de treinamento são considerados ferramentas válidas } \\
\text { para melhorar a segurança. }\end{array}$ \\
\hline $\begin{array}{l}\text { A5- Nurses' knowledge } \\
\text { and practice of vascular } \\
\text { access infection control } \\
\text { in haemodialysis patients } \\
\text { in the Republic of } \\
\text { Ireland. }\end{array}$ & $\begin{array}{l}\text { HIGGINS, M.; EVANS, } \\
\text { D. } 2008 / \text { Journal of } \\
\text { Renal Care/ Irlanda. }{ }^{15}\end{array}$ & $\begin{array}{l}\text { Embora } 92 \% \text { dos entrevistados relataram que políticas tinham } \\
\text { sido desenvolvidas por suas unidades e } 47 \% \text { receberam educação } \\
\text { para controle de infecção no ano anterior, o conhecimento e a } \\
\text { adesão às melhores práticas demonstraram margem significativa } \\
\text { para a melhoria. O estudo recomendou o desenvolvimento de } \\
\text { diretrizes padrão, revisões regulares e atualizações das políticas. }\end{array}$ \\
\hline $\begin{array}{l}\text { A6- Eventos adversos } \\
\text { em hemodiálise: relatos } \\
\text { de profissionais de } \\
\text { enfermagem. }\end{array}$ & $\begin{array}{l}\text { SOUSA, M. et al., 2013/ } \\
\text { Revista de Enfermagem } \\
\text { da USP/Brasil. }{ }^{16}\end{array}$ & $\begin{array}{l}48 \% \text { dos enfermeiros entrevistados não souberam responder } \\
\text { o que era EA. Não houve diferença significativa no número de } \\
\text { eventos relatados entre as diversas categorias profissionais. Muitos } \\
\text { dos relatos foram relacionados ao acesso venoso central, ao } \\
\text { acesso venoso periférico e aos equipamentos e materiais médico- } \\
\text { hospitalares. O estudo contribuiu para a análise crítica quanto } \\
\text { à estrutura e os processos realizados, a qualidade do cuidado } \\
\text { prestado, e estimulou a adoção de medidas para minimizar os EA, } \\
\text { como notificações, mudanças nos processos, entre outras. }\end{array}$ \\
\hline $\begin{array}{l}\text { A7- Infectious } \\
\text { Complications Following } \\
\text { Conversion to Buttonhole } \\
\text { Cannulation of Native } \\
\text { Arteriovenous Fistulas. }\end{array}$ & $\begin{array}{l}\text { LABRIOLA, L. et al., } \\
\text { 2011/ AJKD/ Estados } \\
\text { Unidos. }^{17}\end{array}$ & $\begin{array}{l}\text { O aumento da taxa de infecção não alcançou significância } \\
\text { estatística até o terceiro ano, após a implementação da técnica } \\
\text { de botão. Foi suposto que os enfermeiros, depois de receberem } \\
\text { meticulosa formação inicial, progressivamente prestam menos } \\
\text { atenção ao protocolo de higiene da técnica de botão. A diminuição } \\
\text { da taxa de eventos infecciosos, após a equipe passar por } \\
\text { reeducação sustenta essa hipótese. Outros fatores que poderiam } \\
\text { interferir na taxa de infecções são: o tipo de desinfetante ou } \\
\text { educação do paciente. }\end{array}$ \\
\hline $\begin{array}{l}\text { A8- Complications } \\
\text { observed in older new } \\
\text { haemodialysis patients in } \\
\text { Taiwan. }\end{array}$ & $\begin{array}{l}\text { KUO, F. et al., 2014/ } \\
\text { Australasian Journal on } \\
\text { Ageing/ Taiwan. }{ }^{18}\end{array}$ & $\begin{array}{l}\text { O estudo mostrou que pacientes com idade superior a } 65 \text { anos têm } \\
\text { maior propensão a falhas no acesso vascular e um maior índice de } \\
\text { hospitalizações do que os pacientes mais jovens. Foi evidenciado } \\
\text { que o risco de falhas no acesso vascular e hospitalizações dos } \\
\text { pacientes idosos geram maior custos para os planos de saúde. }\end{array}$ \\
\hline $\begin{array}{l}\text { A9- Surveillance } \\
\text { of dialysis events: } \\
\text { 12-Month experience } \\
\text { at five outpatient adult } \\
\text { hemodialysis centers in } \\
\text { Kuwait. }\end{array}$ & $\begin{array}{l}\text { BADAWY, D.; } \\
\text { MOWAFI, H.; AL- } \\
\text { MOUSA, H., 2014/ } \\
\text { Journal of Infection and } \\
\text { Public Health/ Kuwait. }{ }^{19}\end{array}$ & $\begin{array}{l}\text { Eventos basais em diálise foram significativamente mais comuns } \\
\text { em pacientes com cateteres permanentes e temporários, quando } \\
\text { comparados com pacientes com fístulas e enxerto. A vigilância das } \\
\text { taxas revelou significativamente menor taxa de hospitalização e } \\
\text { hemocultura positiva média, no entanto, taxas significativamente } \\
\text { maiores de início de antimicrobianos intravenosos na população } \\
\text { estudada quando comparadas a dados NHSN publicados. }\end{array}$ \\
\hline $\begin{array}{l}\text { A10- A successful } \\
\text { approach to fall } \\
\text { prevention in an } \\
\text { outpatient hemodialysis } \\
\text { center. }\end{array}$ & $\begin{array}{l}\text { HEUNG, M. et al., } \\
\text { 2010/ Clin J Am Soc } \\
\text { Nephrol/ Estados } \\
\text { Unidos. }^{20}\end{array}$ & $\begin{array}{l}\text { No período de referência, ocorreu o total de } 22 \text { quedas, sendo } 14 \\
\text { envolvendo pacientes e oito funcionários ou visitantes. Análise } \\
\text { de causa raiz identifica deficits educacionais do pessoal e perigos } \\
\text { ambientais como os fatores de risco mais significativos. Depois } \\
\text { de um período de intervenção com formação do pessoal e } \\
\text { modificações ambientais apenas ocorreram três quedas. }\end{array}$ \\
\hline
\end{tabular}




\begin{tabular}{|c|c|c|}
\hline $\begin{array}{l}\text { A11- Sellado de catéteres } \\
\text { con gelafundin versus } \\
\text { heparina sódica. }\end{array}$ & $\begin{array}{l}\text { SIERRA, R., 2010/ } \\
\text { Revista de la Sociedad } \\
\text { Española de Enfermería } \\
\text { Nefrológica/ Chile. }^{21}\end{array}$ & $\begin{array}{l}\text { Na aplicação de ambos os protocolos houve aparição de infecções. } \\
\text { Sendo elas, em sua maioria, locais e foram nas sessões que a } \\
\text { selagem ocorreu com heparina que a maioria das infecções } \\
\text { locais apresentou-se. Já nas infecções sistêmicas, o resultado } \\
\text { foi semelhante em ambos os períodos de estudo, sendo outras } \\
\text { medidas profiláticas necessárias para prevenir esses eventos. Em } \\
\text { ambos os casos o número de intervenções foram aceitáveis para } \\
\text { a disfunção do acesso, não havendo hospitalizações causadas } \\
\text { por esse motivo. Conclui-se que não há contraindicação para o } \\
\text { uso de gelofundina no priming dos ramos do cateter para a sua } \\
\text { preservação. }\end{array}$ \\
\hline $\begin{array}{l}\text { A12-Análisis de los } \\
\text { factores que influyen } \\
\text { en la aparición de } \\
\text { complicaciones y } \\
\text { supervivencia de los } \\
\text { catéteres venosos } \\
\text { centrales para } \\
\text { hemodiálises. }\end{array}$ & $\begin{array}{l}\text { FERNANDEZ, R. et } \\
\text { al., 2014/ Enfermería } \\
\text { Nefrológica/ Espanha. }^{22}\end{array}$ & $\begin{array}{l}\text { Dos } 1.231 \text { cateteres, } 70 \text { tiveram um episódio de infecção. A taxa de } \\
\text { infecção observada (2010-2012) foi de } 0.75 / 1000 \text { dias de cateter; } \\
\text { taxa considerada excelente segundo os critérios que marcam } \\
\text { normas da literatura científica. Isso mostra que o cumprimento } \\
\text { de um rigoroso protocolo que se fundamenta na aplicação de } \\
\text { medidas universais de assepsia é viável para evitar as infecções } \\
\text { sem expor os pacientes aos possíveis efeitos secundários de } \\
\text { qualquer tratamento farmacológico. }\end{array}$ \\
\hline $\begin{array}{l}\text { A13- Nível de seguridade } \\
\text { percebida por el paciente } \\
\text { de hemodiálises crônica. }\end{array}$ & $\begin{array}{l}\text { GUERRERO, V. M. et } \\
\text { al., 2016/ Enfermería } \\
\text { Nefrológica/. } \\
\text { Espanha. }^{23}\end{array}$ & $\begin{array}{l}100 \% \text { dos inquiridos sentem-se seguros durante a sessão de hemo- } \\
\text { diálise. Em termos de educação, } 63 \% \text { gostariam de receber mais } \\
\text { educação, embora } 70 \% \text { digam que sabem como atuar diante de } \\
\text { complicações. Na lista de fatores de estresse, as complicações do } \\
\text { acesso vascular, o tempo conectado à máquina, o transporte e a } \\
\text { temperatura ambiente obtêm pontuações mais altas. Acredita-se } \\
\text { que as intervenções das enfermeiras poderiam ser realizadas para } \\
\text { reduzir os estressores, realizando atividades educativas e recrea- } \\
\text { tivas. }\end{array}$ \\
\hline $\begin{array}{l}\text { A14- La satisfacción del } \\
\text { paciente como compo- } \\
\text { nente de la calidad en las } \\
\text { unidades de diálisis. }\end{array}$ & $\begin{array}{l}\text { TOLEDANO, M. C. L. } \\
\text { et al., 2016/ Enfermería } \\
\text { Nefrológica/ Espanha. }{ }^{24}\end{array}$ & $\begin{array}{l}\text { O estudo inferiu que os pacientes que recebem tratamento de } \\
\text { diálise na Unidade de Gestão Clínica estão muito satisfeitos com } \\
\text { o serviço, com pequenas diferenças, dependendo da unidade que } \\
\text { recebem o tratamento. Foram identificados aspectos de melhorias } \\
\text { no serviço, que estão sendo abordadas para aumentar a satisfação } \\
\text { do paciente e, portanto, a qualidade dos cuidados fornecidos. }\end{array}$ \\
\hline
\end{tabular}

Fonte: Tabela produzida pelas autoras. Salvador, 2017.

Após a leitura dos artigos e sumarização dos mesmos, foram identificadas semelhanças nos conteúdos apresentados, surgindo assim, quatro categorias. Sendo elas: a) Implementação de ações para a segurança do paciente em diálise, b) Clima/Cultura de segurança em pacientes em diálise, c) Conhecimento dos profissionais de saúde sobre segurança do paciente em diálise e $\mathrm{d}$ ) Incidentes/Eventos Adversos em diálise.

\section{Discussão}

No Gráfico 1, apresenta-se a distribuição dos resultados por ano e localidade. É possível observar que o ano de maior produção científica acerca do tema seja 2014, com quatro artigos publicados, seguido dos anos de 2008, 2010, 2011 e 2016 com dois artigos publicados em cada. Já os demais anos apresentados no gráfico têm um artigo publicado. 
Apesar da relevância do tema Segurança do Paciente, e este estar em ênfase desde 2004, com a criação da Aliança Mundial pela Segurança do Paciente e em tratamentos de diálise e a temática ter ganhado notoriedade também em 2004 com a RDC no. 154, nota-se que as produções científicas emergiram apenas em 2008, evidenciando a escassez das mesmas no âmbito nacional e internacional.

Houve uma distribuição homogênea em relação ao local de publicação. Apenas a Espanha se sobressai com três artigos publicados. O idioma inglês é predominante na pesquisa com nove artigos, seguido da língua espanhola com quatro artigos e a língua portuguesa com apenas um artigo. Tal evidência da predominância da língua inglesa pode ser explicada por Ortiz, ${ }^{25}$ que afirma ser o inglês a língua da ciência.

A partir da análise dos conteúdos dos artigos incluídos na revisão, emergiram quatro categorias. A categoria denominada "Implementação de ações para segurança do paciente em diálise" foi evidenciada em quatro artigos (A1, A2, A7, A11), onde três desses artigos (A2, A7, A11) trouxeram ações voltadas para os acessos para hemodiálise visando à diminuição de eventos como perdas sanguíneas, infecções, obstruções e internações hospitalares, e um artigo (A1) trouxe a implementação de uma lista de verificação na promoção da segurança do paciente.

Haynes et al. ${ }^{26}$ afirmam em seu estudo, realizado em oito cidades de diferentes países, que a implementação de um checklist ancorado nas recomendações da Organização Mundial de Saúde (OMS) reduz a mortalidade e a morbidade no pós-operatório. A aplicação do referido checklist para cirurgias formulado pelos autores teve como resultado a diminuição do índice de mortalidade dos pacientes em aproximadamente $47 \%$ e do índice de morbidade em aproximadamente $36 \%$. Demonstrando, assim, que o uso do checklist é um potencial instrumento capaz de reduzir um grande número de mortes e condições debilitantes, já levando em consideração a variedade das circunstâncias econômicas e da diversidade populacional.

Assim, inferimos que a implementação de uma lista de verificação contribui significativamente com a avaliação do serviço prestado, pois além de ser um artifício composto por condutas que devem ser seguidas, possibilita, ainda, a análise e a intervenção em pontos estratégicos de possíveis falhas que podem estar comprometendo a qualidade da assistência prestada. Ressalta-se ainda que, apesar da literatura trazer um grande número de relatos do uso de checklist para cirurgia segura, o dispositivo pode ser adaptado em tratamentos dialíticos, visto a sua complexidade e potencialidade na ocorrência de erros.

Os artigos A2, A7 e A11 apresentam ações para a implementação da segurança do paciente voltadas aos acessos vasculares.

O acesso vascular temporário é estabelecido por meio da inserção de um cateter em uma veia calibrosa. ${ }^{27}$ Já a Fístula Arteriovenosa, tida como um acesso definitivo, consiste na junção de uma artéria com uma veia, fazendo que o sangue arterial dilate a parede das veias e possibilite a inserção de agulhas para a realização do tratamento dialítico. ${ }^{3}$

Os pacientes em uso de cateter estão mais susceptíveis a infecções quando comparados aos pacientes em uso de acesso defi- 
nitivo, sendo a infecção uma das principais causas de mortalidade entre os pacientes renais crônicos. ${ }^{27,28,29}$

Com a comprovação em diversos estudos científicos acerca da maior incidência de infecção em pacientes dialíticos em uso de acesso temporário, o artigo A11 traz ações a serem implementadas para a diminuição de infecção em cateteres como o uso de substâncias na selagem desse acesso.

Bevilacqua et al. ${ }^{30}$ e Bleyer $^{31}$ afirmam que o uso de selos com diversos antibióticos e outras substâncias a exemplo do citrato vêm sendo testadas e comprovadas a sua eficácia na prevenção das infecções quando comparados ao uso da heparina, porém, seu uso deve ser avaliado de forma individual, garantindo menores prejuízos ao paciente.

Os artigos A2 e A7 trazem ações voltadas para a fístula arteriovenosa. Em seu conteúdo, o artigo A2 apresenta um dispositivo desenvolvido para a detecção de extravasamento em fístulas arteriovenosas, intercorrência que o estudo de Silva e Nunes $^{32}$ detectou em maior predominância em pacientes em uso de fístula arteriovenosa para a realização da hemodiálise.

A técnica de buttonhole consiste na punção em sítio constante de uma Fístula Arteriovenosa (FAV) com a formação de um túnel onde a agulha é sempre introduzida. ${ }^{33,34,35}$ Silva e colaboradores ${ }^{33}$ trazem em seu estudo que esse tipo de punção, apesar de proporcionar menos dor, menor erro de punção e hematoma, confere um risco aumentado para a ocorrência de infecções e, por isso, deve ser destinado a pacientes selecionados. O estudo realizado por Castro et $a l .{ }^{34}$ e Silva et al. ${ }^{35}$ não menciona a relação da técnica de buttonhole com o risco de infecções. Já o artigo A7 estuda a possível relação entre infecção e a referida técnica, constatando que a avaliação constante dos indicadores de infecção do serviço e educação continuada entre os pacientes e profissionais pode inferir na diminuição desse evento adverso.

$\mathrm{Na}$ categoria "Clima/cultura de segurança do paciente em diálise", quatro artigos (A3, A4, A13 e A14) convergiram em seus conteúdos. Nos artigos A3 e A4, são evidenciadas as diferenças na percepção do clima de segurança entre diferentes categorias profissionais, e é trazida em consenso a importância da criação de canais de fácil acesso para a comunicação de questões que englobam a segurança do paciente como sendo um artifício para a criação do referido clima de segurança nas unidades de diálise.

Conforme trazem Santiago e Turrini, ${ }^{36}$ os conceitos de clima e cultura organizacional, apesar de serem diferentes, sobrepõem-se, sendo cultura de segurança definida como o produto de valores, atitudes, competências e padrões de comportamentos individuais compartilhados com o grupo, os quais determinam o compromisso, a capacidade e a competência de uma organização segura. ${ }^{37} \mathrm{E}$ clima de segurança, definido como características mensuráveis da cultura organizacional, por meio de percepções e atitudes dos indivíduos, em determinado tempo. ${ }^{38}$

Nos artigos A3 e A4, é identificado que existem diferenças na percepção do clima de segurança e comprometimento com a segurança entre os líderes da organização e os profissionais da área clínica. Sendo que no artigo A3 é trazida a percepção de um sentimento de maior compromisso com a segurança do paciente por parte dos líderes 
da área clínica em detrimento dos líderes da organização. Já em A4, a gestão apresenta maior clima de segurança que o pessoal de linha de frente.

Batalha e Melleiro, ${ }^{39}$ em seu estudo sobre a cultura de segurança do paciente em um hospital de ensino, que teve como um de seus objetivos avaliar a percepção de profissionais de enfermagem acerca da cultura de segurança do paciente, evidenciaram que na dimensão "apoio da gestão hospitalar para a segurança do paciente" houve uma das piores distribuições, possibilitando inferir que a gestão não oferece clima de trabalho que promova a segurança do paciente, demonstrando, então, que a segurança do paciente não é prioridade maior.

Esses achados vão de encontro com o exposto na Portaria $n^{\circ}$. 529, de $1^{\circ}$. de abril de 2013, que afirma que a Cultura de Segurança possui cinco características a serem operacionalizadas pelos gestores da organização, quais sejam: a) cultura de que todos os trabalhadores, incluindo profissionais envolvidos no cuidado e gestores, assumem responsabilidade pela segurança individual, da equipe, dos pacientes e seus familiares; b) cultura que prioriza a segurança acima de metas financeiras e operacionais; c) cultura que encoraja e recompensa a identificação, a notificação e a resolução dos problemas relacionados à segurança; d) cultura que, a partir da ocorrência de incidentes, promove o aprendizado organizacional; e e) cultura que proporciona recursos, estrutura e responsabilização para a manutenção efetiva da segurança. ${ }^{6}$

Diante disso, pode-se afirmar que não deveriam existir diferenças entre o clima de segurança da gestão e o pessoal da linha de frente, visto que gestores devem fomentar essa cultura nos profissionais em prol de um objetivo único que é a assistência segura, em que todos devem estar comprometidos. Trazendo a importância do trabalho em equipe, desenvolvendo atividades que não estejam focadas apenas em controlar ações com função punitiva.

Essa função punitiva da qual a gestão é encarregada pode ser uma das possibilidades da diferenciação no clima de segurança. $O$ que os gestores de instituições de saúde devem incorporar é que os erros não acontecem de forma isolada, podem ser reflexo de falhas ativas, como violação às normas ou de condições latentes como decisões gerenciais. ${ }^{36}$

Ambos os artigos, A3 e A4, trazem como alternativa para sanar essa diferença no clima de segurança melhoria nos meios de comunicação.

A temática dos artigos A13 e A14 assemelhou-se com a proposta da categoria clima/cultura de segurança, proporcionando esse agrupamento, pois conforme definição já aqui presente, o clima de segurança é mensurado a partir da percepção de atitudes e percepções dos indivíduos. E esses artigos trazem a avaliação da satisfação do paciente como medida de avaliação da qualidade do serviço. Essa avaliação permite o fortalecimento da participação dos pacientes nos processos de planejamento e exercício do controle social. A qualidade da técnica empregada nos serviços, a comunicação e a relação entre profissionais/paciente/família, e a opinião dos usuários são fundamentais para a melhoria da atenção ao paciente. ${ }^{40}$

O conteúdo dos artigos A5 e A6 confluíram na apresentação do conhecimento dos profissionais de saúde sobre segurança do 
paciente como objeto de sua pesquisa. Originando, assim, a categoria "Conhecimento dos profissionais de saúde para segurança do paciente em diálise”.

O artigo A6 traz como dado preocupante que quase metade dos profissionais de enfermagem entrevistados não sabia o que é Evento Adverso (EA). O dado é tido como preocupante, visto que os profissionais de enfermagem, assim como obtido no estudo de Dias et al., ${ }^{41}$ são identificados como profissionais fundamentais para a propagação da cultura de segurança.

Sendo a enfermagem classe dominante no quesito quantitativo nas unidades de saúde do Brasil e do mundo, além de serem os profissionais responsáveis por grande parte das ações de cuidado à saúde da população, estando em maior contato com os pacientes, evidencia-se a importância do conhecimento desses profissionais sobre a temática segurança do paciente, considerando que a identificação dos eventos adversos nas etapas do cuidar e do sistema organizacional contribuem para o desenvolvimento de estratégias que promovam a cultura de segurança do paciente. ${ }^{42}$

Portanto, uma vez o profissional não tendo conhecimento do que se trata, de fato, o EA, mostra-se por demais temerosa a sua atuação, indo, assim, de encontro com o Código de Ética dos Profissionais de Enfermagem em seu artigo 16, que diz ser de responsabilidade dos profissionais "assegurar ao cliente uma Assistência de Enfermagem livre de danos decorrentes de imperícia, negligência ou imprudência" ${ }^{43}$ (p. 2).

As consequências que os EA causam são diversas e não somente para os pacientes, mas também para os profissionais, que, após cometerem um erro, podem desenvolver sentimentos ruins, como vergonha, culpa e depressão, influenciando negativamente sobremaneira na sua atuação. Além disso, podemos acrescentar rompimento do vínculo de confiança entre o profissional e o paciente, o incremento de gastos com tratamentos adicionais, prolongamento de internamentos e repercussão negativa na avaliação da qualidade da instituição. ${ }^{44,45}$

Nas unidades de diálise existem fatores que contribuem para a ocorrência de EA devido à sua complexidade, como: utilização de alta tecnologia por meio de equipamentos médico-hospitalares, a exemplo da máquina de hemodiálise e materiais específicos; necessidade de equipe multiprofissional especializada e treinada; realização de procedimentos invasivos; administração de medicamentos, incluindo os potencialmente perigosos; além de alta rotatividade de pacientes devido à execução de várias sessões de hemodiálise por semana. ${ }^{45}$ Logo, tais fatos deixam inferir a necessidade de enfermeiros capacitados, que desenvolvam ações voltadas à identificação de falhas que propiciem EA, conscientizando toda a equipe, bem como, difundindo dentro do serviço a cultura de segurança do paciente.

No artigo A5, é abordado o conhecimento do profissional de enfermagem na prática de controle de infecção de acesso vascular para a hemodiálise, sendo esse um dos principais eventos adversos decorrentes do tratamento dialítico. $3,27,28,46$

Ferreira et al. ${ }^{47}$ em seu estudo objetivaram descrever o perfil de infecção e resistência de cateter de hemodiálise, em que a principal causa das infecções de cateteres de hemodiálise está relacionada à manipulação 
desses dispositivos pela equipe hospitalar e a própria colonização bacteriana no sítio de inserção.

A partir desse achado, podemos afirmar que a falta de conhecimento da equipe de enfermagem acerca das técnicas adequadas para a manipulação desse acesso, assim como a ausência de processos bem desenhados que definam padrões a serem seguidos e de um sistema organizacional voltado para o gerenciamento de riscos propiciam a ocorrência de EA.

$\mathrm{Na}$ categoria "Incidentes/Eventos Adversos em diálise" convergiram em seus conteúdos os artigos A6, A8, A9, A10, A12 que trazem em sua grande maioria incidentes/eventos adversos correlacionando-os ao tipo de acesso vascular. Apenas um artigo (A10) traz como temática as quedas como incidente/evento adverso.

Segundo o Programa Nacional de Segurança do Paciente (2013), incidente é caracterizado como evento ou circunstância que poderia ter resultado, ou resultou, em dano desnecessário ao paciente. Já Evento Adverso é considerado um incidente que resulta em dano ao paciente. ${ }^{6}$

A maioria dos artigos traz referências e associações à ocorrência de eventos adversos ao acesso vascular utilizado para a hemodiálise. Sendo o cateter venoso central um predisponente ao acontecimento desses eventos e as ações citadas para proporcionar maior segurança de o paciente estarem direcionadas a esse tipo de acesso.

Apenas o artigo A10 traz as quedas como evento adverso nas unidades de diálise. O referido artigo identifica os fatores que propiciam as quedas e analisa se as intervenções realizadas, como formação de pessoal e mudanças ambientais interferiram positivamente na diminuição das quedas.

A implementação de protocolos, de indicadores de avaliação e caracterização dos pacientes de risco são ferramentas gerenciais importantes para o enfermeiro no processo de melhoria da qualidade e da segurança na assistência ao paciente. Essa ferramenta permite redirecionar ações de intervenção e sinalizar a necessidade das orientações educacionais aos clientes e à equipe assistencial. ${ }^{48,49}$

Como exemplo da relevância da implementação de protocolos na prevenção de quedas é aprovado pela Portaria no ${ }^{\circ} 2.095$ de 24 de setembro de 2013, o Protocolo de Prevenção de Quedas. Esse protocolo tem como proposta implantar/implementar medidas que avaliem os riscos dos pacientes, assegurando um ambiente seguro para a atuação de um cuidado multiprofissional de qualidade, promovendo a educação do paciente, familiares e profissionais. ${ }^{50}$

Os demais artigos incluídos nessa categoria por semelhança de conteúdo convergiram ao explanarem os acessos venosos temporários como maiores precursores de eventos adversos. Sendo os eventos de maior ocorrência as infecções, seguidos da obstrução dos mesmos. As barreiras citadas para garantir a diminuição dos riscos e com isso a prestação de um serviço de qualidade focado na segurança do paciente foram: incentivos a notificações, mudanças nos processos com uso de protocolos e educação continuada.

O tema Segurança do Paciente na terapia renal substitutiva ganha destaque por intermédio da RDC 154, de 15 de junho de 2004, com a determinação da implantação e im- 
plementação de um Programa de Controle de Infecção e Eventos Adversos (PCIEA). A criação de um Programa nas unidades de diálise, que em sua denominação já dá ênfase ao controle das infecções, faz menção a um de nossos achados, as infecções relacionadas aos acessos para a hemodiálise sendo o mais encontrado no nosso estudo, principalmente aquelas relacionadas ao acesso venoso temporário.

Além das infecções, diante da análise dos estudos incluídos nesta revisão integrativa evidenciou-se que os principais eventos adversos em diálises estavam relacionados à:

- Deslocamento da agulha em punções de FAV - A2

- Infecções relacionadas ao acesso para diálise - A5, A6, A7, A9, A11 e A12

- Disfunção do acesso temporário para diálise - A6, A8, A9, A11, A12

- Coagulação do sistema extracorpóreo - A6, A12

- Quedas-A10

- E que as principais ações presentes nos estudos para a diminuição dos eventos adversos e promoção de um serviço centrado na segurança do paciente foram:

- Educação continuada;

- Definição de diretrizes e políticas;

- Adoção de ações não punitivas com incentivo à notificação dos eventos adversos;

- Implementação de listas de tarefas e responsabilidades;

- Checklist de verificação.

As estratégias educacionais foram as mais apresentadas para o controle da ocorrência de eventos adversos, pois facilitam a adesão do profissional às medidas implementadas e favorecem a criação de uma cultura de segurança. Estratégias gerenciais também são citadas, como a criação de protocolos e diretrizes a serem seguidos, padronizando as ações por meio de conhecimentos científicos e adaptações à realidade da instituição. Outra estratégia mencionada é $o$ incentivo às notificações dos eventos adversos, tendo elas o objetivo de reconhecimento dos riscos e não apenas um caráter punitivo.

Por intermédio dos estudos analisados, podemos inferir que a identificação de riscos e a implementação de ações voltadas às falhas identificadas podem otimizar sobremaneira o tratamento dos renais crônicos, bem como, contribuir com o processo de trabalho dos profissionais. Como consequência disso, senão liquidar, diminuir significativamente as taxas de eventos adversos decorrentes de ações carentes no que tange à segurança, prevenção e administração de intercorrências ocorridas no exercício do tratamento de diálise nos ambientes hospitalares.

\section{Considerações Finais}

As unidades de diálise destacam-se devido à sua complexidade, elevados custos, necessidade de mão de obra qualificada, realização de procedimentos invasivos e aumento cada vez maior de procedimentos devido ao aumento de pacientes com Insuficiência Renal Crônica com necessidade de terapia renal substitutiva. Nesse contexto, essas unidades se revelam como um ambiente propício para o acontecimento de Eventos Adversos, necessitando, assim, da fomentação de estratégias que identifiquem 
e previnam os riscos para o acontecimento desses eventos, propiciando aos pacientes uma assistência livre de danos.

Apesar do tema Segurança do Paciente estar em evidência a nível mundial e a Doença Renal Crônica estar em exponencial crescimento, nota-se uma escassez na produção científica nacional e internacional, destacando a produção nacional com apenas um estudo.

Com o achado predominante neste estudo de que a infecção é o principal evento adverso que acomete pacientes submetidos a tratamentos dialíticos, sendo este prevalecente em pacientes em uso de acesso temporário, podemos afirmar a necessidade do desenvolvimento de estudos com estratégias que viabilizem a diminuição desse evento, seja por meio do uso de substâncias, definição de protocolos e diretrizes e/ou estratégias educacionais. Um instrumento presente na pesquisa que também pode intervir de forma positiva na diminuição dos índices de infecção, atuando como roteiro das condutas que devem ser seguidas, sendo uma estratégia para a realização de avaliações constantes para a implementação de ações em pontos estratégicos, são as listas de verificação. Já utilizadas em outros segmentos da saúde, essas listas devem ser difundidas nos serviços de diálise como mais uma estratégia para a realização de uma assistência de qualidade.

Neste estudo, é perceptível a importância da atuação de todos os profissionais de saúde para a manutenção de um clima de segurança nas instituições de saúde. Para tal atuação, faz-se necessário a prática de gestores e dos trabalhadores operacionais ancorada numa comunicação efetiva, com confian- ça mútua, permitindo ações construtivas e não punitivas, que incentivem a notificação de eventos adversos, utilizando-os como estratégia de aprendizado.

Ainda com relação à atuação dos profissionais de saúde, a falta de conhecimento dos enfermeiros acerca dos eventos adversos é um dado inquietante, visto que são os profissionais em maior quantitativo nas unidades de saúde que prestam assistência direta aos pacientes, propiciando, assim, uma relação de confiança e sendo a atuação dos mesmos utilizada como parâmetro na identificação da qualidade do serviço. Esse é um dos dados obtidos no nosso estudo, que reforça a importância do desenvolvimento de estratégias educacionais, presentes em destaque também nesta revisão, para a manutenção da segurança do paciente em serviços de saúde.

Um indicativo de qualidade do serviço de saúde presente no estudo, mas não tão valorizado em instituições de saúde, é a avaliação da satisfação do paciente. A avaliação desse aspecto é importante, pois se configura como um indício do clima de segurança e uma estratégia importante para a identificação de riscos e planejamento de ações. Além de incorporar o paciente e a família na responsabilidade de um ambiente de saúde seguro.

Um evento adverso ao qual os pacientes em tratamento dialítico estão expostos, no entanto, não apareceu na pesquisa, e é aquele relacionado à qualidade da água utilizada na terapia. Sendo a Tragédia de Caruaru precursora de políticas que vislumbrassem a segurança do paciente em diálise, esse deveria ser um ponto sempre presente quando se trata dessa temática. 
Espera-se que esta pesquisa possa contribuir para a discussão das práticas assistenciais e gerenciais nas unidades de diálise, fomentando a implantação/imple- mentação de estratégias que viabilizem uma assistência de qualidade e livre de danos, vislumbrando a segurança desses pacientes que dependem desse tratamento para a manutenção da vida.

\section{REFERÊNCIAS}

1. Brunner \& Suddarth. Tratado de Enfermagem Médico-cirúrgico, 11 ed. Rio de Janeiro: Guanabara Koogan, 2009.

2. Sesso RC, Lopes AA, Thomé FS, Lugon JR, Martins CT. Inquérito Brasileiro de Diálise Crônica 2016. Jornal Brasileiro de Nefrologia 2017; 39 (3): 261. Disponível: <http://www.jbn.org.br/ details/1962/pt-BR/inquerito-brasileiro-de-diali>.

3. Fermi MRV. Diálise para Enfermagem, 2. ed. Rio de Janeiro: Guanabara Koogan; 2010.

4. Pestana JOM, Freitas TVS, Junior HTS. Transplante Renal: manual prático, 1. ed. São Paulo: Livraria Balieiro, 2014.

5. Westphal GA, Garcia VD, Sousa RL, Franke CA, Vieira KD, Birckholz VRZ et al. Diretrizes para avaliação e validação do potencial doador de órgãos em morte encefálica. Rev Bras Ter Intensiva 2016; 28 (3): 220-255. Disponível: <http://www.scielo.br/pdf/rbti/v28n3/0103-507X-rbti-28-03-0220.pdf $>$.

6. Brasil MS. Portaria no 529 de $\mathbf{1}^{\circ}$. de abril de 2013. Institui o Programa Nacional de Segurança do Paciente (PNSP). Brasília: Ministério da Saúde, 2013.

7. Lemos KCR, Lima FM, Nascimento KS, Lira MN. Cenário atual da Enfermagem em Nefrologia do Recife e Região Metropolitana. Revista de Pesquisa Cuidado é Fundamental Online 2015; 7 (2): 2.349-2.361.

8. Souza M. T., Silva MD, Carvalho R. Revisão Integrativa: o que é e como fazer. Einstein 2010; 8 (1): 102-6. Disponível em: <http://www.scielo.br/scielo.php?pid=\$167945082010000100102\&sc ript $=$ sci_arttext\&tlng $=\mathrm{pt}>$.

9. Mendes KDS, Silveira RCCP, Galvão CM. Revisão Integrativa: método de pesquisa para a incorporação de evidências na saúde e na enfermagem. Texto Contexto Enfermagem 2008; 17 (4): 758-64. Disponível em: <http://www.scielo.br/scielo.php?script=sci_arttext\&pid $=$ S0104-07072008000400018 $>$.

10. Bardin L. Análise de Conteúdo, 70. ed. Lisboa: Geográfica Editora, 2009.

11. Marcelli D., Matos A., Sousa F., Peralta R., Fazendeiro J., Porra A. et al. Implementation of a quality and safety checklist for haemodialysis sessions. Clin Kidney J 2015; 8 (3): 265-270.

12. Ahlmen J., Gydell K., Hadimeri H., Hernandez I., Rogland B., Strombom U. A new safety device for hemodialysis. Journal Compilation 2008; 12: 264-267.

13. Taher S., Hejaili F., Karkar A., Shaheen F., Barahmien M., Al Saran AA. Safety climate in dialysis centers in Saudi Arabia: a multicenter study. Journal of Patient Safety 2014; 10 (2): 101-104.

14. Di Benedetto A., Pelliccia F., Moretti M., d'Orsi W., Starace F., Scatizzi L. et al. What causes an improved safety climate among the staff of a dialysis unit? Report of an evaluation in a large network. Journal of Nephrology 2011; 24 (5): 604-612.

15. Higgins M., Evans DS. Nurses' knowledge and practice of vascular access infection control in haemodialysis patients in the Republic of Ireland. Journal of Renal Care 2008; 34 (2): 48-53.

16. Sousa MRG, Silva AEBC, Bezerra ALQ, Freitas JS, Miasso AI. Eventos adversos em hemodiálise: relatos de profissionais de enfermagem. Revista de Enfermagem da USP 2013; 47 (1). 
17. Labriola L., Crott R., Desmet C., Andre G., Jadoul M. Infectious Complications Following Conversion to Buttonhole Cannulation of Native Arteriovenous Fistulas. AJKD 2011; 57 (3): 442448.

18. Kuo FC, Chiang CL, Lee SY, Wu CJ, Chen HH, Chen YW. Complications observed in older new haemodialysis patients in Taiwan. Australasian Journal on Ageing 2014; 33 (2): 86-92.

19. Badawy DA, Howafi HS, Al-Mousa HH. Surveillance of dialysis events: 12-Month experience at five outpatient adult hemodialysis centers in Kuwait. Journal of Infection and Public Health 2014; 7: 386-391.

20. Heung M., Adamowski T., Segal JH, Malani PN. A successful approach to fall prevention in an outpatient hemodialysis center. Clin J Am Soc Nephrol 2010; 5 (10): 1.775-1.779.

21. Diaz RS. Sellado de catéteres con gelafundina versus heparina sódica. Revista de la Sociedad Española de Enfermería Nefrológica 2010; 13 (4).

22. Fernandez GER, Alvarez MMR, Rodriguez CFP. Análisis de los factores que influyen en la aparición de complicaciones y supervivencia de los catéteres venosos centrales para hemodiálises. Enfermería Nefrológica 2014; 17 (1).

23. Guerrero VM, García GP, Hidalgo CGO, Arribas VM, Peña JR. Nível de seguridade percebida por el paciente de hemodiálises crônica. Enfermería Nefrológica 2016; 19 (3).

24. Toledano MCL, Cantarero IL, López EG, Cuesta RCC, Montero RC. La satisfacción del paciente como componente de la calidad en las unidades de diálisis. Enfermería Nefrológica 2016; 19 (2).

25. Ortiz R. As ciências sociais e o inglês. Revista Brasileira de Ciências Sociais 2004; 19 (54). Disponível em: <http://www.scielo.br/scielo.php?script=sci_arttext\&pid=S0102$-69092004000100001 \& \operatorname{lng}=$ en\&nrm=iso\&tlng=pt>.

26. Haynes AB, Weiaer TG, Berry WR, Lipsitz SR, Breizat AS, Dellinger EP et al. A Surgical Safety Checklist to Reduce Morbidity and Mortality in a Global Population. The New England Journal of Medicine 2009; 360: 491-499. Disponível em: <http://www.nejm.org/doi/pdf/10.1056/ NEJMsa0810119>.

27. Daugirdas JT, Blacke PG, Ing TS. Manual de Diálise, 4. ed. Rio de Janeiro: Guanabara Koogan, 2008.

28. Barbosa DA, Gunji CK, Bittencourt ARC, Belasco AGS, Diccini S et al. Co-morbidade e mortalidade de pacientes em início de diálise. Acta Paulista de Enfermagem 2006; 19 (3). Disponível em: <http://www.scielo.br/scielo.php?pid=S010321002006000300008\&script=sci_ abstract\&tlng=pt $>$.

29. Terra FS, Costa AMDD, Figueredo ET, Morais AM, Costa MD, Costa RD et al. As principais complicações apresentadas pelos pacientes renais crônicos durante as sessões de hemodiálise. Revista Brasileira de Clínica Médica 2010; 8 (3): 187-192. Disponível em: <http://files.bvs.br/ upload/S/1679-1010/2010/v8n3/a001.pdf>.

30. Bevilacqua JL, Gomes JG, Santos VFB, Canziani, MEF. Comparação entre citrato trissódico e heparina como solução para selo de cateter em pacientes em hemodiálise. J Bras Nefrol 2011; 33 (1): 86-92. Disponível: <http://www.scielo.br/pdf/jbn/v33n1/v33n1a12.pdf >.

31. Bleyer AJ. Use of Antimicrobial Catheter Lock Solutions to Prevent Catheter - Related Bacteremia. Clin J Am Soc Nephrol 2007; 2: 1.073-1.078. Disponível: <http://cjasn.asnjournals.org/ content/2/5/1073.full>.

32. Silva KA, Nunes ZB. As intervenções de enfermagem mais prevalentes em um serviço de hemodiálise frente às intercorrências com a fístula arteriovenosa durante a sessão de hemodiálise. J Health Sci Inst 2011; 29 (2): 110-113. Disponível: <https://www.unip.br/comunicacao/publicacoes/ics/edicoes/2011/02_abr-jun/V29_n2_2011_p110-113.pdf >. 
33. Silva GST, Silva RA, Nicolino AM, Pavanetti LC, Alasmar VL, Guzzardi R. et al. Experiência inicial com técnica de buttonhole em um centro de hemodiálise brasileiro. Jornal Brasileiro de Nefrologia 2010; 32 (3). Disponível: <http://www.scielo.br/scielo.php?script=sci_arttext\&pi $\mathrm{d}=$ S0101-28002010000300006>.

34. Castro MCM, Silva CF, Souza JMR, Assis MCSB, Aoki MVS, Xagoraris M. et al. Punção da fístula arteriovenosa com a técnica em casa de botão com agulha romba. Jornal Brasileiro de Nefrologia 2010; 32 (3). Disponível em: <http://www.scielo.br/scielo.php?script=sci_arttext\&p id $=$ S0101-28002010000300010>.

35. Silva DM, Gurgel JL, Escudeiro CL, Ferreira HC. Satisfação dos pacientes com a técnica de buttonhole. Cogitare Enfermagem 2015; 20 (3): 482-486. Disponível: <http://docs.bvsalud.org/ biblioref/2016/08/1156/41576-162977-1-pb.pdf>.

36. Santiago THR, Turrini RNT. Cultura e clima organizacional para segurança do paciente em Unidades de Terapia Intensiva. Revista da Escola de Enfermagem da USP 2015; 49 (esp.): 123-130. Disponível: <http://www.scielo.br/pdf/reeusp/v49nspe/1980-220X-reeusp-49-spe-0123.pdf>.

37. Reis CT, Laguardia J., Martins M. Adaptação transcultural da versão brasileira do Hospital Survey on Patient Safety Culture: etapa inicial. Cad Saúde Pública 2012; 28 (11): 2.199-2.210. Disponível: < http://www.scielo.br/pdf/csp/v28n11/19.pdf>.

38. Santiago THR, Turrini RNT. Cultura e clima organizacional para segurança do paciente em Unidades de Terapia Intensiva [letter]. Revista da Escola de Enfermagem da USP 2015; 49 (esp.): 123-130. Colla JB, Bracken AC, Kinney LM, Weeks WB. Measuring patient safety climate: a review of surveys [abstract]. Qual Saf Health Care 2005; 14 (5): 364-6.

39. Batalha EMSS, Melleiro MM. Cultura de segurança do paciente em um hospital de ensino: diferenças de percepção existentes nos diferentes cenários dessa instituição. Texto Contexto Enfermagem 2015; 24 (2): 432-44. Disponível: <http://www.scielo.br/pdf/tce/v24n2/pt_0104-0707tce-24-02-00432.pdf>.

40. Coutinho NPS, Tavares MCH. Atenção ao paciente renal crônico, em hemodiálise, sob a ótica do usuário. Cad Saúde Coletiva 2011; 28 (11): 232-239. Disponível: <http://www.iesc.ufrj.br/ cadernos/images/csc/2011_2/artigos/csc_v19n2_232-239.pdf>.

41. Dias JD, Mekaro KS, Tibes CMS, Mascarenhas SHZ. The nurses' understanding about patient safety and medication erros. Rev Min Enferm 2014; 18 (4): 874-880.

42. Tobias GC, Bezerra ALQ, Moreira IA, Paranaguá TTB, Silva AEBC. Conhecimento dos enfermeiros sobre a cultura de segurança do paciente em Hospital Universitário. Revista de Enfermagem UFPE Online 2016; 10 (3): 1.071-9. Disponível: <http://www.revista.ufpe.br/revistaenfermagem/index.php/revista/article/view/8615/pdf_9868>.

43. COFEN - Resolução COFEN nº. 311/2007: Código de Ética dos Profissionais de Enfermagem. Disponível: <http://novo.portalcofen.gov.br/wp-content/uploads/2012/03/resolucao_311_anexo.pdf>.

44. Vicent C. Segurança do paciente: orientações para evitar eventos adversos. São Paulo: Yendis Editora, 2009.

45. Sousa, MRG, Silva AEBC, Bezerra ALQ, Freitas JS, Miasso AI. Eventos Adversos em hemodiálise: relatos de profissionais de enfermagem. Revista de Enfermagem da USP 2013, 47 (1): 76-83. Disponível em: <http://www.scielo.br/pdf/reeusp/v47n1/a10v47n1.pdf >.

46. Machado GRG, Pinhati FR. Tratamento de diálise em pacientes com insuficiência renal crônica. Cadernos UniFOA 2014, 26: 137-148. Disponível em: <http://web.unifoa.edu.br/cadernos/ edicao/26/137-148.pdf>.

47. Ferreira ACB, Deprá MM, Pies OTC, Sousa ICR, Rocha LKM, Filho JCPS. Infecções em cateter de hemodiálise: aspectos microbiológicos e de resistência em uma unidade de referência em 
uma unidade de referência em Belém. Revista Sociedade Brasileira de Clínica Médica 2014; 12(4). Disponível em: <http://files.bvs.br/upload/S/1679-1010/2014/v12n4/a4372.pdf>.

48. Correa AD, Marques IAB, Martinez MC, Laurino PS, Leão ER, Chimetão DMN. Implantação de um protocolo para gerenciamento de quedas em hospital: resultados de quatro anos de seguimento. Revista Escola de Enfermagem USP 2012; 46 (1): 67-74. Disponível em: <http://www. scielo.br/scielo.php?script=sci_arttext\&pid=S0080-62342012000100009>.

49. Paiva MCM, Paiva SAR, Berti HW, Campana AO. Caracterização das quedas de pacientes segundo notificação em boletins de eventos adversos. Revista Escola de Enfermagem USP, 2010; 44 (1): 134-8. Disponível em: <http://www.scielo.br/scielo.php?script=sci_arttext\&pid $=$ S0080-62342010000100019>.

50. Brasil MS. Portaria n⿳0. 2.095 de 24 de setembro de 2013. Aprova os protocolos básicos de Segurança do Paciente. Brasília: Ministério da Saúde, 2013.

\section{DADO(S) AUTOR(RES)}

\section{MARIA ROSA SILVA LEMOS}

Residente em Nefrologia pela Universidade do Estado da Bahia - UNEB. Enfermeira pela Universidade Federal do Recôncavo da Bahia - UFRB. Universidade do Estado da Bahia (UNEB), Salvador/ BH - Brasil.mariarosa.lemos@yahoo.com.br

\section{EDENISE MARIA SANTOS DA SILVA BATALHA}

Enfermeira, Doutoranda da Escola de Enfermagem da Universidade de São Paulo. Professora Auxiliar do Departamento de Ciências da Vida da Universidade do Estado da Bahia (UNEB), Salvador/ $\mathrm{BH}$ - Brasil. edenisemaria@gmail.com

Submissão em: 29/11/2017

Aceito em: 05/01/2018 